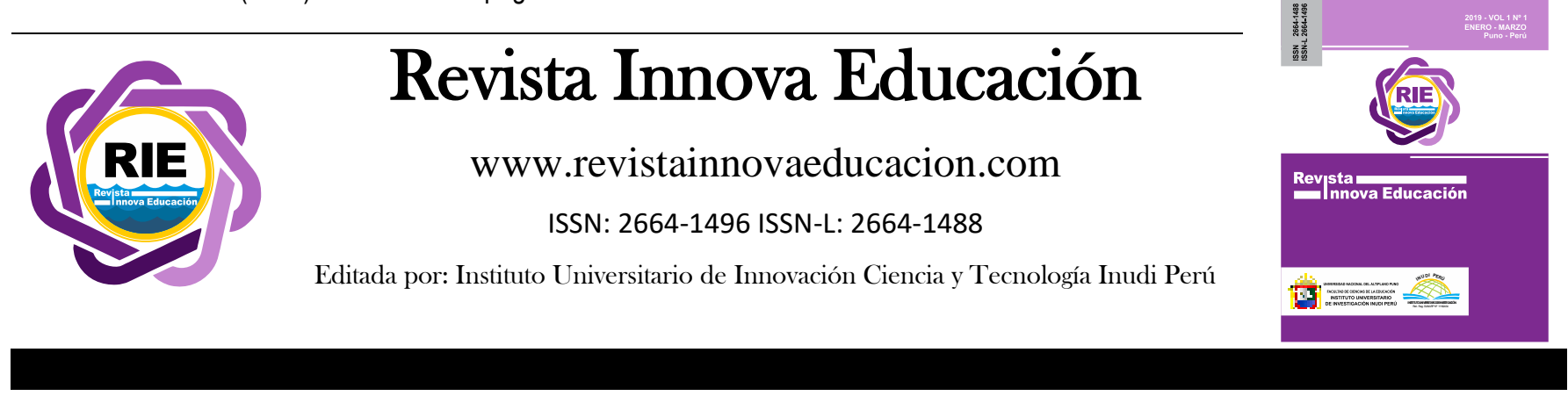

\title{
La alfabetización matemática en profesionales universitarios: imposibilidad o re-significación urgente
}

\author{
Mathematical literacy in university professionals: impossibility or urgent re-significance \\ Milagros Rodríguez ${ }^{1}$ \\ Universidad de Oriente, Cumaná-Estado de Sucre, Venezuela \\ http://orcid.org/0000-0002-0311-1705
}

DOI: https://doi.org/10.35622/j.rie.2020.02.001

Recibido 27/01/2020/ Aceptado 11/04/2020 Publicado 26/04/2020

\begin{tabular}{ll}
\hline ARTíCULO ORIGINAL & El presente artículo estudia la alfabetización matemática en los profesionales \\
universitarios como imposibilidad o re-significación urgente desde las voces de \\
PALARAS CLAVE & $\begin{array}{l}\text { diferentes profesionales. En la investigación se realizó entrevistas a nueve (9) } \\
\text { profesionales de diferentes áreas. Mediante una indagación cualitativa, un estudio }\end{array}$ \\
alfabetización, & exploratorio, deviniendo con ello categorías emergentes. Los resultados revelan los \\
matemática, re- & análisis de las encuestas categorías emergentes como: democratización de la \\
significar, & matemática; pereza intelectual en profesionales; malas políticas educativas en \\
profesionales, & Venezuela; falsas creencias, emociones negativas hacia la matemática e imposición de \\
universitarios & una matemática reduccionista en el aula y el encuentro de los saberes científicos de la \\
& matemática y los saberes soterrados de la misma. En conclusión, la alfabetización \\
& matemática tiene actualmente en las profesionales líneas de salida como: el profesional \\
promotor de la matemática como ciencia legada de la humanidad, como \\
responsabilidad ciudadana de cada uno; minimización en comunidades de aprendizaje \\
de las falsas creencias de la matemática y su aprendizaje, todos podemos; la \\
democratización de la matemática y las políticas educativas re-significadas a favor de \\
la promoción de la ciencia legado de la humanidad. Nos incita a pensar que la \\
problemática y luego la necesidad hoy más que nunca de la alfabetización matemática \\
es responsabilidad de todos.
\end{tabular}

KEYWORDS

mathematical, literacy, re-signifying, university, professionals

\begin{abstract}
This article studies mathematical literacy in university professionals as an impossibility or urgent re-significance from the voices of different professionals. In the investigation, interviews were conducted with nine (9) professionals from different areas. Through a qualitative inquiry, an exploratory study, thereby becoming emergent categories. The results reveal the analysis of the emerging categories surveys such as: democratization of mathematics; intellectual laziness in professionals; bad educational policies in Venezuela; false beliefs, negative emotions towards mathematics and imposition of a reductionist mathematics in the classroom and the meeting of the scientific knowledge of mathematics and the underground knowledge of it. In conclusion, mathematical literacy currently has in the professional exit lines such as: the professional promoter
\end{abstract}

\footnotetext{
${ }^{1}$ Correspondencia: milagros.elena.rodriguez@gmail.com
} 
of mathematics as a legacy science of humanity, as the citizen responsibility of each one; minimization in communities of learning of the false beliefs of mathematics and its learning, we can all; the democratization of mathematics and re-signified educational policies in favor of the promotion of the legacy science of humanity. It encourages us to think that the problem and then the need today more than ever for mathematical literacy is everyone's responsibility.

Cuando estudio matemáticas mis pensamientos se elevan hasta el universo, y sus similitudes de perfección con este me hacen entender el poema más bello que de la misma vida se hace; creo vibrar en ese abrigar que cuando puedo estar en la estasis más hermosa al sentir tu Espíritu Santo. Milagros Elena Rodríguez (2018: 40)

\section{INTROITO, ESTADO DEL ARTE, CRISIS, JUSTIFICACIÓN Y TEORÍAS}

No quiero saber nada de números, la verdad estudie mi carrera para no saber nada de las matemáticas; estas son palabras frecuentemente pronunciados en profesionales que manifiestan abiertamente su ignorancia por lo más elemental de la matemática, y por su aprendizaje.

La actitud matemática que indica la confianza en el uso de la matemática, el interés, curiosidad e inventiva al hacer matemáticas y valorar la aplicación matemática en la experiencia diaria es escasa en muchos profesionales universitarios que intencional o no, propagan tal realidad en sus vidas y aumentan con ello las falsas creencias de lo que realmente es la matemática, su enseñanza y aprendizaje. La desfachatez en pleno de profesionales que gozan del desarrollo científico con grandes aportes de la matemática y desmitifican su utilidad.

En general, la matemática es una ciencia cuyos resultados se usan en el contexto cotidiano de cualquier persona; en el caso de los profesionales universitarios no existe un área del conocimiento que no tenga relación o una profunda transdisciplinar con la matemática, se puede decir que un profesional que no posea los conocimientos básicos o competencias esenciales de la matemática no puede ejercer adecuadamente, su razonamiento critico abstracto no es competente con la alta complejidad necesaria para estos tiempos de competitividad y globalización.

De la transdisciplinariedad, el Artículo 4 de los 15 artículos que componen la Carta de la transdisciplinariedad firmada en Arrábida afirma que "la clave de la bóveda de la transdisciplinariedad reside en la unificación semántica y operativa de las acepciones a través y más allá de las disciplinas" (Basab, 1996, p.25).

Ahora, "la alfabetización o cultura digital de todos los individuos resulta indispensable no sólo en los procesos de identificar dilemas o problemas disciplinarios, sino también en la búsqueda de información, representación, exploración, solución de problema y comunicación de resultados" (Santos, 2015, p.145). 
Consideramos acá la alfabetización un concepto complejo que nos sólo atiende a competencias y habilidades; sino el pensar complejamente, por tanto, ayuda, en especial, en la relación educativa, unir, reunir, construir y relacionar los conocimientos, estos están en constante cambio, tal como lo explica Morín (1994).

En el caso de la matemática partiendo de una concepción compleja, la Educación Matemática debe, de acuerdo con Rodríguez (2019) contribuir, a través de un contenido matemático, a la formación y desarrollo integral de las personas, procurando que se involucren profundamente en su propia formación, motivándolas y estimulándolas con el propósito de que se eduquen como tales, y no como si se tratase de construir y programar maquinas en serie, lo que con frecuencia se olvida en las clases de matemáticas, ejerciendo un mecanismo de poder que incrementa cada vez más el rechazo y predisposición por dicha ciencia.

Entonces esta postura anterior, siendo compleja impide ver al educando, que las características del conocimiento matemático son especiales, pues permiten que puedan dirigirse al fomento de la creatividad, pensamiento crítico, capacidad de aprender de modo autónomo lo que los estudiantes necesiten en y para la vida, y de aplicar estas nociones para resolver los problemas que la vida les plantee. Todas excelencias del ser humano pertenecen a su diversidad y su condición humana; todo ello es posible en la enseñanza de la matemática adecuada y pertinente, Morín (2006).

Es así como, partiendo que la Educación Matemática, la alfabetización y con ello la alfabetización matemática son procesos complejos, "la alfabetización matemática se configura mediante un repertorio básico de conocimientos, técnicas y destrezas matemáticas a las que hay que añadir un cúmulo de capacidades y competencias constituidas sobre la base de la utilización de dicho repertorio en contextos cotidianos" (González, 2018, p.11).

De la experiencia de la autora, en mi contacto con la propia alfabetización urgente de la matemática, en muchas charlas, conferencias me he conseguido opiniones de matemáticos como: es hora de trabajar en orientar todo lo que hemos investigado para el desarrollo de nuestro país, así como también encontrar nuestros propios resultados y no sólo depender de un artículo referencial, es tarea de todos socializar la importancia de la matemática en lo cotidiano en el saber popular, en el desarrollo del ser humano y para ello debemos estudiar su origen y su estructura, para poder entender su lenguaje y poder aplicarlo.

Las consecuencias de ser analfabeto en la matemática, es de una gravedad extrema, en los profesionales universitarios en insólito; lo que dice que los currículos y formación no está acorde a la transdisciplinariedad de los conocimientos de su carrera con la matemática, no conociendo la matemática, o muy poco, el "lector común" es excluido. Si trata de entender el significado del 
argumento científico probablemente se equivocará o entenderá algo que no es. Esto es también cierto, pero es una verdad que se encuentra a mitad de camino a la indolencia” (Fehr, 2011, p. 69).

En el Programa para la Evaluación Internacional de Estudiantes (PISA), la Organización para la Cooperación y el Desarrollo Económicos (OECD), designa el concepto de alfabetización en matemáticas como la capacidad "para identificar y comprender el papel que juegan las matemáticas en el mundo, plantear juicios matemáticos bien fundamentados e involucrarse en las matemáticas, según lo requiera una persona en su vida actual y futura como un ciudadano constructivo, preocupado, reflexivo" (OECD, 2003, p.3).

Es clara la definición anterior; si los profesionales universitarios estuvieran ubicados, y los que lo están, en el papel esencial de la ciencia legado de la humanidad en el mundo y en cada una de sus carreras, con una huella clara en el mundo; si decidieran asumir tal realidad colaborarían en la alfabetización urgente y necesaria de la matemática en la vida de sus estudiantes, en general y de las personas que atienden en sus tareas. Además, tomado en cuanta del papel ciudadano que tienen ante el mundo.

La democratización de la matemática, desde luego concepto relacionado con la alfabetización de la matemática, la educación con jóvenes y adultos debería formar parte de "un esfuerzo por paliar las desigualdades en el acceso a oportunidades educativas en pro de una sociedad que favorezca la democratización del conocimiento; cualquiera y en cualquier sentido, teniendo en cuenta lo que las personas saben y quieren saber" (Palma, 2018, p.111). Esta democratización colaboraría a cambiar la visión de la matemática y en un futuro a evitar la pereza intelectual del ciudadano o el descuidado interés por la matemática.

La pereza intelectual por la matemática, impide que los profesionales desarrollen y tenga en si las competencias esenciales, llevan así la ciencia al aula de la manera que ellos fueron formados; siguen considerándola difícil inalcanzable y con ello han desvalorizado la ciencia legado de la humanidad. De ello, se trata acá al averiguar en profesionales de diferentes carreras el interés y por qué. Nótese que esta pereza intelectual muchas veces se promueve constantemente por el rechazo hacia la matemática; pues la mayoría conoce de la importancia de la ciencia y su aplicabilidad. Aun así, sigue desmitificando la matemática. Por ello, en un estudio exploratorio en esta investigación se analizan las opiniones y con la respuesta de la pregunta: ¿A qué se debe que las necesidades matemáticas elementales no estén cubiertas en profesionales universitarios (de diferentes áreas); por el contrario, conscientes de la importancia de las matemáticas siguen promoviendo su rechazo? La explicabilidad de la metodología se hace en el siguiente apartado.

La matemática para todos es cada vez más urgente, no se trata de sólo científicos de la ciencia legado de la humanidad sino portadores de habilidades y razonamientos que apliquen en 
sus oficios y cotidianidad. Se trata de una dimensión política de la matemática al servicio de la humanidad que no podemos dejarle a cargo sólo de los matemáticos.

Rico (2007) resalta como la matemática esta por todos lados, en cualquier proceso; explicita que cada fenómeno natural es una manifestación del cambio; el mundo en nuestro entorno muestra una multitud de relaciones temporales y permanentes entre fenómenos. Los organismos cuando crecen y sus cambios, los ciclos de las estaciones, el flujo y reflujo de las mareas, los ciclos de empleo y desempleo, los cambios climáticos y los cambios en los indicadores económicos. Algunos de los procesos de cambio pueden ser descritos y modelados directamente mediante funciones matemáticas: lineales, exponenciales, periódicas, discretas o continuas. Las relaciones matemáticas tienen usualmente la forma de ecuaciones o de desigualdades, pero también se presentan relaciones de naturaleza más general. El cuerpo humano y su funcionalidad, en ritmos, procesos y perfección son patrones matemáticos.

Todo lo anterior y más tienen su contenido matemático que debe ser intereses de todos, conocido en su generalidad: Nos sorprende que alguien no conozca lo elemental de historia de sus países, de la división de sus estados y parroquias del lenguaje materno. De la misma manera debe sorprendernos que los procesos matemáticos elementales de la vida diaria, del hacer cotidiano sean ignorado; si promovidos sus rechazos por profesionales que teniendo adversidad a la matemática, por sus razones personales no superadas promuevan el miedo y rechazo hacia la ciencia responsable en el medio donde se desenvuelven y en sus propias familias.

La idea de alfabetización matemática, la capacidad de un individuo de formular, utilizar e interpretar "las matemáticas en una variedad de contextos. Incluye razonar de manera matemática y utilizar conceptos, procedimientos, hechos y herramientas de esa disciplina para describir, explicar y pronosticar fenómenos" (OCDE, 2013, p.25).

Podemos devenir en la crisis en la enseñanza de la matemática que todavía sigue siendo mostrada como ejercicio de poder en el aula, ya ha venido señalando Rodríguez (2018) la enseñanza es un arte que implica interacciones íntimas con otros, donde los sentimientos, emociones estados de ánimo, el yo y la vida de los actores del proceso educativo están íntimamente unidos a su labor educativa; la enseñanza de la matemática no está desprovista de esta realidad. La sociedad entera debe comprender la urgente necesidad de mostrar la matemática viva, consustanciada con los procesos dialógicos en la vida del ser humano.

Sin embargo, el estado del arte de la problemática de la enseñanza de la matemática sigue en crisis; explicita Rodríguez (2019) que en el aula suceden situaciones narrativas de imposición: ¡yo explico y ustedes copian, repitan así el procedimiento, no deben cambiar nada; ese algoritmo es así y muchos lo repiten! Son unos pocos los que han llegado a aprenderlo, pues les encanta 
cambiar las cosas y confundirse. Las estadísticas de aprobados son muy bajas. Y de repente un silencio en el lugar, se termina la hora de clase y hasta acá la comunicación hoy en la clase de matemáticas. Son relatos tantas veces repetidos en los procesos de enseñanza de la matemática; aún en plena época tecnológica.

De esta manera aún en estos tiempos seguimos concluyendo que el fracaso escolar en matemáticas se ha vuelto perseverante, "y con él la potencial exclusión de muchos estudiantes de unirse al deseo por las matemáticas. ¡Es como si el deseo de expandir el alcance de más matemáticas para todos generara mecanismos de selección y exclusión de muchos!" (Valero, 2017, p.90). Con las consecuencias ya emitidas las matemáticas siguen promoviéndose sólo a unos pocos denominados inteligentes.

Los riesgos y costos de que cada vez seamos más analfabetos en matemáticas, han sido largamente estudiados, en 2018 la Revista Semana analizaba el tema; expresando que "si bien el analfabetismo matemático dificulta la vida diaria de las personas, sus consecuencias son también globales. Muchos estudios sugieren una correlación entre la carencia de habilidades aritméticas con el desempleo nacional, la productividad e incluso la salud física".

En lo que sigue para el desarrollo de la investigación se da: la metodología, objetivo, instrumento de investigación, categorías y desarrollo de la investigación; esta última que es la alfabetización matemática en profesionales en las voces de los entrevistados, el análisis de los encuestados ante la interrogante planteada inicialmente y finalmente las conclusiones en el camino que incitan a continuar estudiando el problema.

\section{MÉTODO, OBJETIVO, CATEGORÍAS Y DESARROLLO DE LA INVESTIGACIÓN}

El objetivo de la investigación fue estudiar la alfabetización matemática en los profesionales universitarios como imposibilidad o re-significación urgente desde las voces de diferentes profesionales y el análisis de categorías emergentes que de sus opiniones emanan.

En la investigación se realizó entrevistas a nueve (9) profesionales de diferentes áreas; todos profesionales universitarios: docentes de matemática; matemático, físico, psicólogo, químico, contador. El instrumento usado fue una entrevista no estructurada, que no necesita validación. El criterio de elección fue libre, que cumplieran con la esencia de ser de diferentes profesiones universitarios y que estuvieran dispuestos a responder la única interrogante abierta que se especifica a continuación.

Mediante una indagación cualitativa, en un estudio exploratorio se analizan las opiniones y con la respuesta de la pregunta: ¿A qué se debe que las necesidades matemáticas elementales no 
estén cubiertas en profesionales universitarios (de diferentes áreas); por el contrario, conscientes de la importancia de las matemáticas siguen promoviendo su rechazo? Una vez extraída las ideas más resaltantes que aportan a la temática: alfabetización matemática en los profesionales universitarios, se ubicarán sus aportes en categorías emergentes que devienen de sus opiniones. Y más delante de acuerdo con ello se dan salidas y aportes sustantivos a la crisis. No se aplica el análisis fenomenológico interpretativo (AFI) sino que interpretando las opiniones de los profesionales se extraen categorías que constituyen el objeto de estudio.

Algunas categorías emergentes de la alfabetización matemática desde las voces de los entrevistados se dan a continuación, analizando sus ideas.

\section{La alfabetización matemática en profesionales: las voces de los entrevistados}

Ante la pregunta: ¿A qué se debe que las habilidades y conceptos matemáticas elementales no estén cubiertas en profesionales universitarios; por el contrario consientes de la importancia de las matemáticas siguen promoviendo su rechazo? Se seleccionan a continuación ideas capitales de cada entrevistado, ubicadas en la categoría que emerge de sus aportes. Con estas citas de cada entrevistado se hacen análisis y se ubican en categorías emergentes.

\section{Categoría emergente: democratización de la matemática}

El entrevistado 1, matemático, afirma: "es hora de trabajar en orientar todo lo que hemos investigado para el desarrollo de nuestro país, así como también encontrar nuestros propios resultados y no sólo depender de un artículo referencial, es tarea de todos socializar la importancia de la matemática en lo cotidiano en el saber popular, en el desarrollo del ser humano y para ello debemos estudiar su origen y su estructura, para poder entender su lenguaje y poder aplicarlo".

Más que una crítica a la situación y crisis de la alfabetización matemática, el entrevistado 1 aporta la categoría: la democratización matemática que corresponde a los especialistas del área. Reconoce la matemática para científicos como matemáticos, abstracta e indispensable; pero va a la reinvención de dicha ciencia en la vida cotidiana del ser humano para entender la ciencia como lenguaje y utilidad en el desenvolvimiento y la aplicabilidad en las necesidades prioritarias y cotidianas del ser humano.

El ser humano formado universitariamente debe poseer competencias y habilidades que mostrando su vida ciudadana como retrataría del mundo, aportando unas soluciones y manejándose como ciudadano del mundo a quién le debe apostes. No puede el profesional estar de espalda a los problemas del mundo en tanto deviene de él y se debe a él. 


\section{Categoría emergente: pereza intelectual en profesionales}

El entrevistado 2, profesional de la enseñanza de la matemática opina que: "pudiera decir muchas cosas, pero se debe a nosotros mismos (...) Pues resulta más sencillo no esforzarse, excusarse con no tener habilidades, magnificar a los que si dedican, que a pensar (...) Damos por sentado de que no las necesitaremos, con sumar y restar es suficiente (...) ¿Total que tanto? siempre nos ha engañado el del supermercado, el de la bodega por un simple cálculo o un descuento, el gobierno con sus absurdos aumentos (...) y así pasaremos el resto de nuestra vida, con lo que merecemos, literalmente por flojos, por sentirnos tan poquita cosa que más vale robar al otro que honestamente aprender matemáticas y con su uso, ayudarnos".

La pereza intelectual está presente actualmente, pese a la tecnología, a la globalización y a la expansión de las posibilidades para llegar al conocimiento. El conformismo instaurado en nuestras mentes, bien sea porque aprendimos que la matemática es para situaciones específicas, que no podemos aprenderla; o que existen especialistas para resolver los problemas.

Desde el medio que nos desenvolvemos, incluyendo las instituciones educativas hemos mantenido aparte la matemática, desvirtuado su utilidad y hasta la aritmética elemental la realizan sólo con medios electrónicos. Obviando que la matemática también es desarrollo de pensamiento crítico, abstracción, decisión adecuada; enriquecimiento de nuestro lenguaje. Entre tantos sustantivos que pudiéramos transdisciplinarmente aporta a nuestra formación y dejar de separar las ciencias en general de las matemáticas y tratarla como instrumento de utilidad y no de aprehensión de su valor.

\section{Categoría emergente: malas políticas educativas en Venezuela}

El entrevistado 3, profesional universitario de la contaduría afirma: "pienso que las necesidades elementales de matemáticas que no estén cubierta por profesionales es por culpa de los gobiernos que hemos tenido en Venezuela, ya que he visto que por palanca política un bachiller le dan un cargo tan importante, porque desde primarias se obtienen las bases para entender matemáticas. No estoy de acuerdo que se siga promoviendo el rechazo y menos por los profesionales en el área (...) en el caso de profesionales, si ellos rechazan las matemáticas lo hacen eso será porque no les gusta"

Es delicado estudio, y tal vez necesite la investigación la continuidad en otra investigación para profundizar como en el país, Venezuela, en estos momentos como la matemática como ciencia esencial esta desmitificada en los currículos nuevos de las universidades creadas recientemente; 
en carreras como la ingeniería se observa que la enseñanza de la matemática deja mucho que desear.

Las políticas gubernamentales no han conseguido elevar el nivel académico de nuestra educación pública, entre otras razones porque se carece de una correcta discriminación del cuerpo docente de nuestras instituciones y se reclutan docentes de cualquier área por motivos politiqueros donde juega un papel importante la insolvencia de docentes por la emigración y los pobres salarios; en muchas instituciones universitarias por no decir todas, no existen departamentos de matemáticas como estructuras que desempeñan un papel concluyente en la planificación de la enseñanza de la matemática y en su calidad. Las investigaciones de los profesores de matemáticas no son aplicadas a su práctica, es más no se investigan sus propias acciones en el aula, y con ello su mejora y avance; la opinión del discente no ha importado en la reconstrucción en el aula del docente.

En la Educación Básica en muchas ocasiones no son los especialistas, ni los formados para enseñar matemáticas los encargados de tan loable tarea. La formación de docentes de matemática, en Venezuela, "la comunidad de educadores matemáticos, cada vez realizan mayores aportes con las investigaciones que se realizan desde los núcleos y centros de investigación y los estudios postgraduados que funcionan en las universidades, pero cuyos resultados, lamentablemente no llegan a las instancias ministeriales correspondientes, precisamente por esa falta de vinculación que corta cualquier posibilidad de extrapolar sus hallazgos hacia el gran conglomerado de la educación primaria y media” (León, 2016, p.455).

De igual manera, las malas políticas educativas venezolanas que repercuten en la enseñanza de la matemática en todos los niveles, afirma Cordero (2019) que una de las derivaciones de la falta de efectividad de las políticas educativas venezolanas es la profundización de los problemas estructurales del sistema educativo venezolano; así como la formación permanente de los docentes; las nuevas tendencias, en aprendizaje colaborativo transdisciplinar, entre otros.

Sigue avalando Cordero (2019) avalando la complejidad y transdisciplinariedad como categorías constituyentes de la investigación, que se debe transitar desde la complejidad como sistema de educación fluida, con la integración de contextos educativos y no educativos, con participantes directos e indirectos del sector educativo venezolano. Para finalizar, de acuerdo con Cordero (2019), aún no hay consenso de cómo canalizar las directrices de las políticas educativas ante la polaridad del nuevo milenio de lo estable y lo inestable.

Categoría emergente: falsas creencias, emociones negativas hacia la matemática e imposición de una matemática reduccionista en el aula 
El entrevistado 4, profesional universitario joven matemático afirma que "pienso que el rechazo es generado por miedo a lo desconocido. Al conversar con personas de bachillerato, manifiestan que no saben qué están haciendo. Pareciera que el sistema está diseñado de una forma nemotécnica, de tal manera que solo sepas aplicar el algoritmo, no se discuten los resultados más allá del número. Cuestión que en la universidad puede cambiar, pero ya el tabú está instaurado. Si queremos que una idea sea aceptada o tomada a consideración entre muchos, ésta debe explicarse de forma tal que pueda ser entendida por el receptor común".

En este caso el siguiente entrevistado coincide en la categoría actual. El entrevistado 5, docente doctor en educación opina que "todavía se mantiene la creencia que las matemáticas son para personas inteligentes, que su estudio es demasiado difícil y complicada".

Sigue expresando el entrevistado 5 que "el estudio de las matemáticas genera ansiedad, nervios y angustia por las ideas tan erradas que muchos profesionales tienen. Igualmente, observo mala preparación e incluso en algunos docentes del área".

El entrevistado 6 afirma que "considero que el rechazo a las matemáticas es inculcado desde la edad temprana, bien sea en el hogar o en la escuela. En el hogar por ignorancia, cuando te dicen que sumar y restar es suficiente y en la escuela porque usan la matemática como castigo, "por portarte mal debes hacer los números de uno en uno hasta el 2000".

El entrevistado 7 afirma que "quizás sea la manera de abordar las matemáticas desde un punto de visto muy rígido a la hora de enseñarla, en general los estudiantes suelen tener mejor rendimiento en las materias que le gustan, de alguna manera se ha perdido en interés y por ende se le resta la importancia que merece".

Observe que los entrevistados 4, 5, 6 y 7, a groso modo resumen la escaza alfabetización matemática en profesionales a las falsas creencias, emociones negativas hacia la matemática e imposición de una matemática reduccionista en el aula. Las creencias y actitudes hacia las matemáticas han sido estudiadas hace bastante tiempo. En Rodríguez, Velásquez y Lemus (2019) la dimensión emocional debe ser esencial en el aprendizaje matemático, y se puede aproximar al tema desde las perspectivas psicológicas y sociológicas. Las relaciones entre la dimensión emocional y la matemática no son fáciles y requieren que el docente se prepare específicamente en aspectos concernientes a las áreas de psicología y sociología de la Educación Matemática.

El Estado y la sociedad entera deben colaborar en develar ese velo, sombra negativa que se ha sembrado sobre las personas y la ciencia patrimonio de la humanidad, la matemática. Es hora de que todos colaboren en el sentido de atender que la matemática es creación humana y que el ser humano la puede aprender, recrear y engrandecer con las demás ciencias en unas matemáticas fractálicas que se complejizan con otras áreas del saber donde el ser humano ha llegado. Desde 
luego hay un velo de misterio de la matemática en la naturaleza y en la creación de la humanidad que es de merecido estudio, tanto que se ha llegado a concluir que Dios es matemático. Merecido honor.

Es de resaltar que en esta categoría emergente las políticas educativas en Venezuela no están diseñadas para minimizar las falsas creencias y la forma mecánica de enseñar matemáticas. Tema que debe ser atacado por especialistas, a fin de democratizar la matemática en las aulas y en la calle, en la vida del ciudadano. También un llamado a que la publicidad en general por cualquier medio globalizado, deje de usar la matemática para ejemplificar lo difícil lo inalcanzable; debe ser motivo de vergüenza lucrarse de las creaciones de la humanidad en la que el aporte de la matemática es esencial; y seguir promoviendo la matemática como absurda, difícil e inalcanzable.

\section{Categoría emergente: El encuentro de los saberes científicos de la matemática y los saberes soterrados de la misma}

El entrevistado 8, matemático con 27 años de experiencia, educador e innovador y especialista en cultura, afirma que: "es lamentable la ignorancia promovida por profesionales del rechazo a la matemática; que ha afectado el proceso de enseñanza, su difusión y culturización en cada persona. Urge combinar en la calle, en el hábitat popular en las comunidades de científicos de manera general lo que se sabe de la matemática básica y reconocerla en el arte, en los patrimonios culturales; el uso de estos para promover la alfabetización de la matemática viva, consustanciada con las personas, el cultor y cada ser humano. Los saberes populares soterrados de la matemática deben ser legitimados por los científicos y estos reconocidos en lo popular. Hace falta voluntad política".

La matemática modernista, carente de ética regularizadora, también los es del aporte urgente de los saberes soterrados y la cotidianidad; la cultura está allí en cada uno de sus pueblos, es un espacio rico de aportes educativos a ser tomados en cuenta de manera sustantiva, cuestión que sustenta Rodríguez (2010).

Para el entendimiento de la significancia de los saberes soterrados se desea ir al rescate de aquellos saberes sometidos que fueron descalificados, obviado por las metodologías cientificistas, considerados inacabados, sin carácter científico comprobable como lo es la cultura, la cotidianidad, aquellos como los saberes históricos, ingenuos, considerados de bajo nivel, los saberes soterrados, aprisionados por una filosofía castrante de la matemática, los saberes sometidos son una "serie de saberes calificados como incompetentes, o, insuficientemente elaborados: saberes ingenuos, inferiores jerárquicamente al nivel del conocimiento de la 
cientificidad exigida (...) de estos saberes locales de la gente, de estos saberes descalificados como se ha operado la crítica" (Foucault, 1986, p.5).

Los saberes legos de la matemática, los culturales; los del hábitat popular, de los grupos étnicos son rescatados en su estudio por la Etnomatemática, línea de investigación que tiene resultados importantes que incitan a otra manera de hacer matemática que atiende la matemática cotidiana del hacer del grupo de discentes. La etnomatemática propone una educación que: "estimule el desenvolvimiento de la creatividad desinhibida, conduciendo a nuevas formas de relaciones interculturales e intraculturales (...) hacer de la Matemática una disciplina que preserve la diversidad y elimine la desigualdad discriminatoria (...). El Programa Etnomatemática tiene ese objetivo mayor" (D'Ambrosio: 2006, p. 52)

Así mismo, referido a matemáticos se debe tomar en cuenta que investigar desde la parcela de las disciplinas desliga y contradice la responsabilidad social del docente como ciudadano y la esencia misma de ser humano. Se debe ir sin duda más allá de la disciplina, engrandeciéndola y transversalizandola a los saberes soterrados, aquellos que no pasan por el filo de las ciencias.

\section{Categoría emergente: Mala formación académica}

El entrevistado 9, afirma que "en primer lugar puedo decir que en nuestras universidades los estudiantes que egresan de ellas no están preparados debidamente por falta de profesionales en esa área, esto es un motivo importante para promover el rechazo y no darles la importancia a las matemáticas en proceso de enseñanza- aprendizaje".

Aquí hay un tema de dilatado cuidado: ¿Por qué tenemos docentes con tanta problemática a la hora de enseñar matemática? Es una pregunta de delicado cuidado; posiblemente justificación para otra indagación. En general esa educación colonial, impuesta desde 1492, con la invasión a nuestro región; en especial soslayaron la enseñanza de la matemática en este lado del mundo y de las consecuencias de la invasión a este Continente se sabe que "las primeras olas de colonización europea de los siglos XIV al XVIII trajeron nuevos idiomas, religiones y órdenes sociales que atropellaron a las culturas indígenas, la nueva colonización global impone también nuevas maneras de vivir, de producir y de pensar" (Skovsmose y Valero, 2012, p.27). Y la enseñanza de la matemática no escapo en su hacer, pensar y poder.

En el caso de la ciencia matemática, D’Ambrosio (1996) concibe la ciencia, incluidas las matemáticas, como actores de esta invasión cultural; y, desde luego, la Educación Matemática no es un espectador inocente de los hechos. Ha sido utilizada la ciencia legado de la humanidad para 
la colonización y soslayación de nuestro propio conocimiento de la matemática que los propios originarios han venido construyendo.

La enseñanza de la matemática, no escapa primero de la colonización y luego de la colonialidad; por ello en el Sur emergen movimientos, investigadores que reconociendo los conocimientos y saberes matemáticos propios dan cuenta del impulso y de la valía de los nuestros, de la lucha por hacer lo propio, de develar nuestros aportes a la ciencia legado de la humanidad. La matemática como legado de la humanidad no es parcela de Occidente, ni de nadie; es un derecho, inclusión de todos. Actualmente la enseñanza de la matemática reduccionista, aislada de los procesos dialógicos de los discentes persiste; aunado a un aumento creciente del rechazo a la ciencia, y en Venezuela pese a muchos avances se mantienen las malas políticas educativas soslayadoras de la educación en general.

\section{RESULTADOS: ANÁLISIS DE LOS ENCUESTADOS ANTE LA INTERROGANTE PLANTEADA INICIALMENTE}

En PISA 2000 se evalúa la alfabetización en matemáticas como un todo, en el que siempre se da una combinación de las dimensiones: 1) las habilidades para desarrollar procesos matemáticos, 2) los contenidos matemáticos, 3) los contextos en los cuales se usan las matemáticas. De acuerdo a las opiniones de los encuestados, en la interrogante inicial: ¿a qué se debe que las habilidades y conceptos matemáticas elementales no estén cubiertas en profesionales universitarios?; por el contrario, conscientes de la importancia de las matemáticas siguen promoviendo su rechazo. Aportamos la primera línea de salida: profesional promotor de la matemática como ciencia legado de la humanidad, como responsabilidad ciudadana de cada uno.

Dejando la falsa idea que la matemática es para unos pocos, tal vez llamados anormales, inteligentes. Se trata como lo señalan Rodríguez, Velásquez y Lemus (2019) las matemáticas no son para personas “anormales"; es más no creemos en anormalidades para aprender; todas las personas son distintas, sus procesos, sí creemos que dicha ciencia es para apasionados por el misterio, por lo oculto, por el análisis profundo y crítico de lo que ocurre de la naturaleza, por lo problemas; y que esa pasión se pude despertar; muchos han sido marcados positivamente por grandes docentes, maestros apasionados. La matemática es poesía, es belleza. No hay un hecho creado por el ser humano y en la naturaleza que no lleve la marca de las teorías matemáticas. La matemática como conocimiento posible es un desafío en las mentes de quienes creemos en nosotros mismos, en nuestro poder mental, emocional. Es un misterio inalcanzable el hecho de que quienes se adentran en el mundo de las matemáticas jamás abandona el deseo de saber, de 
crear y de entender. Las matemáticas en la naturaleza son magníficas creaciones de nuestro amado Dios.

Por todo ello y más, la matemática requiere del amor y el reconocimiento de su legado. Necesitan ser rescatada. Este rescate y salvaguarda debe ser responsabilidad de cualquier profesional que conociendo la matemática para el ejercicio de cualquier conocimiento en una profundad transdisciplinar de las ciencias sea promotor de la matemática.

La línea de salida: minimización en comunidades de aprendizaje de las falsas creencias de la matemática y su aprendizaje, todos podemos; la democratización de la matemática. En Rodríguez, Velásquez y Lemus (2019) se afirma que la aversión y las creencias hacia la ciencia legado de la humanidad deben ser atacadas desde los niveles iniciales, desde el juego que atrapa al discente a bajar del pedestal a la matemática y consustanciarla con sus vidas. Y hacer ver que, sin importar la definición de la palabra anormal, en general los entrevistados propenden que las matemáticas son para todas las personas que se dediquen con amor y pasión provocados por maestros y docentes innovadores al mostrar la matemática viva, con mente, cuerpo y corazón en la vida de sus estudiantes.

Es de rescatar que pese a las creencias y rechazos hacia las matemáticas, provocados en la mayoría de las veces por docentes castradores de su esencia, se rescata que la matemática no es una ciencia elitista reservada para superdotados o para personas con una dedicación enfermiza y escasa o nula vida social; la matemática es divertida, esto es; su estudio alegra el espíritu y puede constituirse en un sano esparcimiento para interactuar con nuestros seres queridos y el desenvolvimiento de un ciudadano formado consonó a los nuevos tiempos.

De la categoría emergente el abrazo de los saberes soterrados y científicos de la matemática se deviene la línea de salida: la culturización e inculturación de la matemática con la participación de todos los profesionales y las comunidades. Es de reconocerse que: ¡está en juego en estos precisos instantes el legado de la matemática, se debe despertar del letargo de ver cada día más lejana esta ciencia del ser humano, cuando siendo cada vez más necesaria en la vida de este! Un dilema que se debe dilucidar a fin de cumplir con el objetivo primordial de la matemática como ciencia. Se nota que esta perspectiva, con que despunta la matemática; destinada hacia las necesidades del hombre, es decir, en su parte humana, divina, intelectual, artística, entre otros; la ciencia innegablemente necesaria, y pertenece a la vida cotidiana, y satisfacción de los estamentos antrópicas.

Otra línea de salida para la alfabetización de la matemática es: las políticas educativas resignificadas a favor de la promoción de la ciencia legado de la humanidad. Tenemos que deliberar la enseñanza de las matemáticas, así como "la organización misma de la educación, de la escuela, 
de la administración escolar, del currículo, del lugar del docente y de los alumnos, entre otros. Se trata de reconstruir un imaginario y una identidad desde otro horizonte civilizatorio" (Solano, 2015, p.123). Por ello, en primer lugar, la develación y conocimiento de la condición humana en el discente y el aportar salidas a las falsas creencias y actitudes hacia el aprendizaje de las matemáticas, son urgentes.

La formación del ciudadano como el capital intelectual más importante a gestionar debe ser el objetivo de la enseñanza de la matemática actual; pero no cualquier formación; sino aquella que tome en cuenta "la regeneración moral que necesita la integración, en nuestra propia conciencia y personalidad, de los preceptos de la autoética, a fin de reactivar nuestras potencialidades altruistas y comunitarias” (Morín, 2006, p. 194). Es volverse hacia la formación continua, hacía la humanización, que sólo el ser humano puede construir; aportes que respalda Rodríguez (2014).

Para desarrollar y llevar a la práctica una enseñanza de la matemática re-significada es necesaria la formación de los docentes que orienten una Educación Matemática, para las comunidades, los niños y niñas, los pueblos indígenas dada la complejidad de esta formación (interculturalidad, lenguas, territorios indígenas); para ello el currículo complejo, transdisciplinar y abierto; sumamente reflexivo es urgente. Y con ello la reformulación de los currículos de todas las carreras.

Aunque el tema de currículo no es de profundización en la indagación, es de hacer notar que según González (2008) este diseño curricular mencionado lleva elementos de autoorganización, orden-desorden e incertidumbre, autorreflexión, estrategia compleja, trabajo en equipo (grupos transdisciplinares) y una formación basada en la investigación compleja. Es así como caduca el docente solitario que no intercambia con grupos o comunidades de aprendizaje y deja fuera los elementos culturales, y actividades recreativas o extraacadémicas o de fiestas especiales. Se transversalizan los saberes matemáticos patrimoniales legitimados por los docentes y la cotidianidad del discente.

\section{A MODO DE INICIO Y CONCLUSIONES DE APERTURAS}

En relación a la alfabetización matemática en profesionales universitarios como imposibilidad o re-significación urgente; sin duda es necesario una re-significación urgente de lo que debe ser la alfabetización matemática desde las políticas de estado; promotoras de una culturización e inculturación de la matemática como vía para el desarrollo de los países y de la vida del ser humano. Pero también una tarea de las políticas educativas desde todos los niveles de educación. 
Una tarea titánica en las comunidades; con un amor profundo en cada aspecto cultural y patrimonios cargados de la esencia matemática que debe ser explorada y difundidas. Las investigaciones particulares similares no abundan, en tanto realizadas en profesionales. Más si en estudiantes, su alfabetización entre otras. Las referencias clásicas de la alfabetización matemática son esenciales en el estudio, como las de PISA.

En las instituciones educativas, de acuerdo con Rodríguez (2019) es urgente recuperar una relación educativa dinámica, la autoestima de los discentes, y también con ella la autoestima del docente como profesional, como una manifestación importante en el transcurso de la enseñanza de la matemática e importante para su desarrollo, debido a que se involucra en la configuración de la personalidad, con las relaciones sociales, con su propia afectividad, con sus patrones culturales, con la motivación y el rendimiento en la ciencia; por tanto, se relaciona con su experiencia de vida en su integralidad.

Para recuperar la autoestima, que muchas veces se ha perdido en las clases de matemáticas, en el sentido de pensar que no pueden aprehenderla; es pertinente la creatividad, innovación e imaginación en el acto educativo, de allí que los ambientes de aprendizajes, la colaboración, los estilos de aprendizaje cobran sentido en una educación renovada, innovadora y esperanzadora.

La recuperación de la autoestima y las creencias y afectividad positivas hacia la matemática en profesionales universitarios puede desmitificar esa denominada pereza intelectual que conllevaría a la transdisciplinariedad en la formación de profesionales que no sólo atiendan sus disciplinas sino que conjuguen la importancia de los saberes y estén consiente de ser portadores de conceptos principales de la matemática que le ayudarán en sus carreras, en su desenvolvimiento en la vida. La matemática es indispensable, y pese a ello algunos entrevistados reconociendo la pereza y la falta de interés por ella.

Todo esto no es más, que un reclamo justo que la matemática como reina y auxilio indispensable de todas las ciencias merece y reclama, además que necesita para salvar su legado. Esta visión compleja que también se tendría de la ciencia en cuestión hace entonces que su transdisciplinariedad, historia y filosofía formen parte del educador y esté pueda mediante un dialogo verdadero, reflexivo y un negociar de reglas poner en el escenario de la clase.

En esta investigación se estudió la alfabetización matemática en los profesionales universitarios como imposibilidad o re-significación urgente desde las voces de diferentes profesionales. En la investigación se realizaron entrevistas a nueve (9) profesionales de diferentes áreas, y las conclusiones referentes concuerdan con las del marco teórico que se ha desarrollado, en la indagación cualitativa en particular; la autora es doliente de la problemática, participe de ella; 
es víctima del proceso modernista de formación; pero que se inscribe en ser agente de cambio que estudia y promociona la matemática con cuerpo, mente y corazón.

De los colaboradores entrevistados las líneas de salidas: el profesional promotor de la matemática como ciencia legado de la humanidad, como responsabilidad ciudadana de cada uno; minimización en comunidades de aprendizaje de las falsas creencias de la matemática y su aprendizaje, todos podemos; la democratización de la matemática y las políticas educativas resignificadas a favor de la promoción de la ciencia legado de la humanidad. Nos incita a pensar que la problemática y luego la necesidad hoy más que nunca de la alfabetización matemática es responsabilidad de todos.

En estas líneas de salidas de los entrevistados se dan espacios a la necesidad de alfabetización de la matemática en profesionales universitarios como re-significación urgente, la imposibilidad se minimiza en tanto que se concientice el hecho de que la matemática es esencia y posibilidad de avances de los países; la democratización de la matemática y las políticas educativas deben estar a favor de dicha re-significación.

La trandisciplinariedad en la formación de profesionales debe ser un arma que rompa con la pereza, con el desinterés, las falsas creencias y desmitifique la matemática en la vida de los profesionales; pero también la invitación a los profesionales de las diferentes áreas a indagar sobre los conceptos esenciales de la matemática; a valerse de ella como instrumentos en su hacer, y al disminuir su pereza ante la ciencia. Todo ello disminuiría el colaborar al rechazo de la ciencia. Es de concluir que la alfabetización de la matemática es una tarea por re-significar y que no es imposible; más aún en profesionales.

La crisis en los profesionales que aúnan grandemente en el rechazo y desconocimiento de los aspectos más elementales de la matemática, deviene de una vieja cultura que atañe a las falsas políticas gubernamentales y educativas donde merma cada vez, más, y es el caso especial de Venezuela del estudio de la ciencia legado de la humanidad. Todo a favor de un facilismo y una pereza mental. Es de resaltar que los aportes de la matemática en materia del desarrollo del país y de una ciudadanía formada en valores, elementales aportes de la matemática son una necesidad; bajo la conciencia que sin la transmisión del legado de la matemática no es posible la profundización de conocimiento o saberes algunos.

\section{AGRADECIMIENTOS}

Al grupo de profesionales universitarios que, de manera auténtica, desde la trinchera de su hacer, aceptaron ser entrevistados y respondieron en un fin común: la realización de la investigación. 
Al Magister Scientiarum en Ingles, Johnattan Chimaras, Instituto Tecnológico, Santiago de Chile, por la traducción completa al inglés del artículo. Eternamente agradecida del tiempo dedicado con mucho cariño a ello.

\section{REFERENCIAS BIBLIOGRÁFICAS}

Basarab, N. (1996). La Transdisciplinariedad: Manifiesto. Mónaco: Ediciones Du Rocher.

Cordero, Y. (2019). Constructo teórico de los desafíos de las políticas educativas para Venezuela en el siglo XXI. Una mirada desde el paradigma de Modernidad Líquida. Valencia: Universidad de Carabobo. Tesis de Doctorado.

D’Ambrosio, U. (1996). Educacão matemática: Da teoria à pràtica. Campinas: Papirus.

D'Ambrosio, U. (2006). Ethnomathematics. Link between traditions and modernity. Rotterdam: Sense Publishers.

Fehr, H. (2011). Hacia la alfabetización matemática. Cuadernos de Investigación y Formación en Educación Matemática, 6(7): pp. 57-70.

Foucault, M. (1986). "Por qué estudiar el poder: la cuestión del sujeto". En: AA.VV., Materiales de sociología crítica. Madrid: Ediciones La Piqueta.

González, J. (2008). Reflexiones iniciales sobre la concepción del diseño y desarrollo curricular en un mundo contemporáneo y complejo. Revista Integra Educativa, 1(2): pp.13-58.

González, M. (2018). Competencias básicas en educación matemática. Didáctica de la Matemática Didáctica de la Matemática. Universidad de Málaga. http://114.red-88-12-10.staticip.rimatde.net/mochila/sec/monograficos_sec/ccbb_ceppriego/mates/aspgenerales/Competencias _basicas_en_Educacion_Matematica\%20Gonzalez\%20Mari.pdf

León, N. (2016). La formación del docente de Matemática en Venezuela: una acción en tiempo progresivo. Cuadernos de Investigación y Formación en Educación Matemática. 2016. Año 11. Número 15. pp 443-457. Costa Rica.

Morín, E. (1994). Introducción al pensamiento complejo. Barcelona: Gedisa.

Morín, E. (2006). El método VI. Ética. Madrid: Ediciones Cátedra.

OCDE (2013). PISA 2012 Assessment and Analytical Framework: Mathematics, Reading, Science, Problem Solving and Financial Literacy, Paris, OCDE. Recuperado de: http://dx.doi.org/10.1787/9789264190511-en

OCDE (2013). PISA 2012 Results: Excellence through Equity: Giving Every Student the Chance to Succeed (Volume II). Paris: OCDE.

Palmas, S. (2018). La tecnología digital como herramienta para la democratización de ideas matemáticas poderosas. Revista Colombiana de Educación, (74), pp.109-132.

Revista Semana (2018). Los riesgos y costos de que cada vez seamos más analfabetos en matemáticas. Recuperado de: https://www.semana.com/educacion/articulo/por-quesomos-analfabetos-en-matematicas/576418

Rico, L. (2007). La competencia matemática en PISA. PNA, 1(2): pp.47-66. 
Rodríguez, M, Velásquez, A, y Lemus, J. (2019). ¿Las matemáticas son para “anormales”? Vivencias y sentires de los actores del proceso educativo. Visión Educativa IUNAES, 13(28): pp.73-79.

Rodríguez, M. (2010). Matemática, cotidianidad y pedagogía integral: elementos epistemológicos en la relación ciencia-vida, en el clima cultural del presente. Caracas: Universidad Experimental Politécnica de la Fuerza Armada Nacional. Tesis de Doctorado.

Rodríguez, M. (2014). La gestión del conocimiento en las instituciones educativas en el clima cultural del presente. Praxis Educativa ReDIE, 10: pp.7-27.

Rodríguez, M. (2018). Narrativa de las matemáticas del amor y la amistad: Didáctica poéticas en la enseñanza. Praxis Educativa ReDIE Revista Electrónica de la Red Durango de Investigadores Educativos, 19: pp. 40-55.

Rodríguez, M. (2019). La relación educativa desde la pedagogía integral en la matemática: una innovación por re-significar. Revista Magazine de las Ciencias, 4(4): pp. 12-25.

Santos, M. (2015). Uso coordinado de tecnologías digitales y competencias esenciales en la educación matemática del siglo XXI. En: La educación matemática en el siglo XXI, Xicoténcatl Martínez Ruiz / Patricia Camarena Gallardo Coordinadores, Colección PAIDEIA Siglo XXI: Zacatenco, pp.133-154.

Skovsmose, O. y Valero, P. (2012). Acceso democrático a ideas matemáticas poderosas. En Valero, P. y Ole Skovsmose, O. Educación Matemática crítica. Una visión sociopolítica del aprendizaje y la enseñanza de las matemáticas (pp. 25-64). Colombia: Universidad de los Andes, Centro de Investigación y Formación en Educación.

Solano, J. (2015). Descolonizar la educación o el desafío de recorrer un camino diferente. Revista Electrónica Educare, 19(1): pp.117-129.

Valero, P. (2017). El deseo de acceso y equidad en la educación matemática. Revista Colombiana de Educación, 73: pp.97-126

\section{Agradecimientos / Acknowledgments:}

Expreso mi agradecimiento a los docentes del Departamento de Matemáticas de la Universidad de Oriente, Venezuela.

Conflicto de intereses / Competing interests:

La autora declara que no incurre en conflictos de intereses.

Rol de los autores / Authors Roles:

No aplica.

\section{Fuentes de financiamiento / Funding:}

La autora declara que no recibió un fondo específico para esta investigación.

Aspectos éticos / legales; Ethics / legals:

La autora declara no haber incurrido en aspectos antiéticos, ni haber omitido aspectos legales en la realización de la investigación. 